\title{
An Object Distance Measuring Method of Monocular Vision Mobile Robot
}

\author{
Zhengguang Zheng ${ }^{1}$, Xiaohong Ren ${ }^{1,2^{*}}$ and Yajun Cheng ${ }^{1}$ \\ ${ }^{1}$ Sichuan University of Science and Engineering, Zigong 643000, China \\ ${ }^{2}$ Artificial intelligence Key Laboratory of Sichuan Province, \\ Zigong 643000, China \\ rx88@163.com
}

\begin{abstract}
To raise monocular vision mobile robot perception to the environment and positioning precision, the article put forward a monocular vision distance measuring method based on sub pixel image processing. First select two different images for the same scene obtained from the CCD in different positions as the target images in the process of the mobile robot motion, and then use Canny edge detection and the sub-pixel image ShiTomasi corner extraction and RANSAC feature point matching method to do target image processing. Finally, through analysis of the imaging position change of the same feature point in two images and combining with the motion parameters of the mobile robot itself, the relative position information between the mobile robot and the target could be obtained by calculation for a map generation and path planning for the robot. By the proposed method the positioning experiment in different locations of the targets was carried out for the mobile robot. The results show that the method can meet the mobile robot's request for the timeliness of the control and effectively improve the mobile robot obstacle avoidance and target positioning accuracy.
\end{abstract}

Keywords: monocular vision; sub pixel; distance measurement; mobile robot

\section{Introduction}

All of visual mobile robots require the ability to have environmental awareness, map creation, and path planning, all of which require measurement of environmental depth information. The environment information acquisition based on visual image form has been applied in the field of driver-less and unmanned production workshops. Due to the environmental uncertainty and complexity, ensuring the safety of mobile robots in real time is the first task of the single vision perception and the promise to complete the robot's further intelligence.

Aiming to the problem of general monocular measurement of low precision and timeliness, the monocular vision model sub-pixel image processing based on improved and simplified monocular measurement model, and through the sub-pixel edge corner extraction and matching, combined with the motion parameters of mobile robot obtain the target depth information can locate the obstacles and target.

Numerous researchers have carried out considerable investigations on the monocular vision ranging measurement methods. The dynamic measurement method based on monocular vision is proposed in literature [1], can effectively calculate the target distance, but when access to the blurred images, the quality of target feature point detection and matching would be poor, leading to decreased accuracy obviously; On the other hand, the discrete pixels are used to represent consecutive target points, and the resulting errors are not reduced. A Monocular measurement method proposed in literature [2], applies only to

Received (December 31, 2017), Review Result (February 5, 2018), Accepted (February 14, 2018) 
the fixed camera, and for the application on the mobile robot, the camera will have some bumps along the way, the camera parameters would be changed, so the measurement accuracy will be reduced; Literature [3], it is to calculate the distance between the lens and the object according to the image the magnification and the moving distance of the lens. The lens imaging graphics zoom range is limited. when the image blurred, the target magnification is not easy to measure and calculation; Literature [4] according to the relationship between the focal length of camera and image sharpness to range of objects, but the camera focal length is extremely difficult to solve in real-time, so the method also is not easy to use; The [5][6][7] documents using monocular vision measuring method based on geometric constraints. The method is small computation and real-time performance, but not accurate enough. Literature [8] using a fixed distance double ball as orientation markers, it implemented the monocular vision location system of indoor automatic vehicle, but its application range is some restrictions. The reference [9] according to the target pixel changes ranging between different images, however it only discussed the objective and parallel planes.

\section{Target Feature Point Extraction}

We have a color image which contains sufficient information. For example, Figure 1. Demanding on the processor configuration to color images is larger than gray scale. It consumes more time to process images, not easy to meet the real-time performance of the robot automatic collision avoidance and route planning. So images of calculation are conducted on the gray scale. Using the gray scale images, without losing image detail characteristics, at the same time, it can accelerate the image processing speed.

To filter the gray image obtained, suppress noise in as much as possible under the condition of to retain the image details of the target image. Here using a median filter. It is a typical nonlinear smoothing filter technique. This method can remove impulse noise and the salt-pepper noise while preserving the edge details of images, and the processing results of the gray scale and median filtering of the image are given in Figure 2.

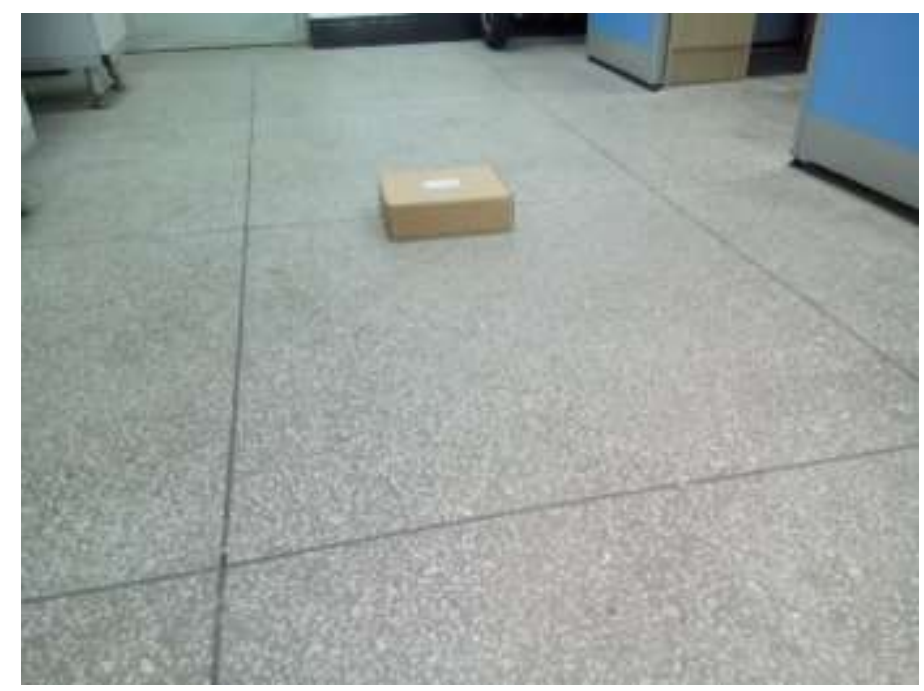

Figure 1. Original Image 


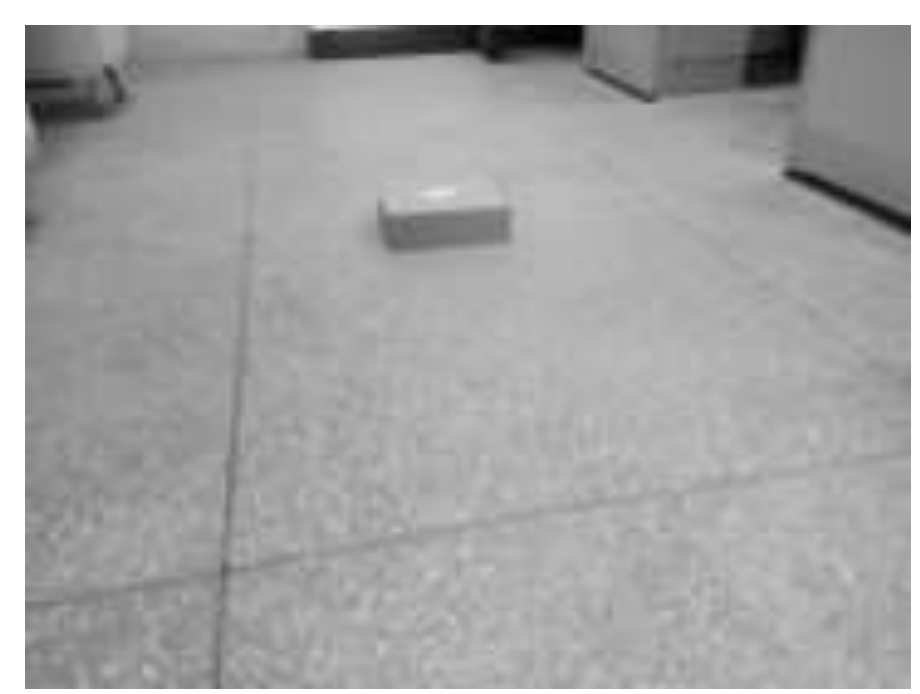

Figure 2. Gray Image and Median Filtering

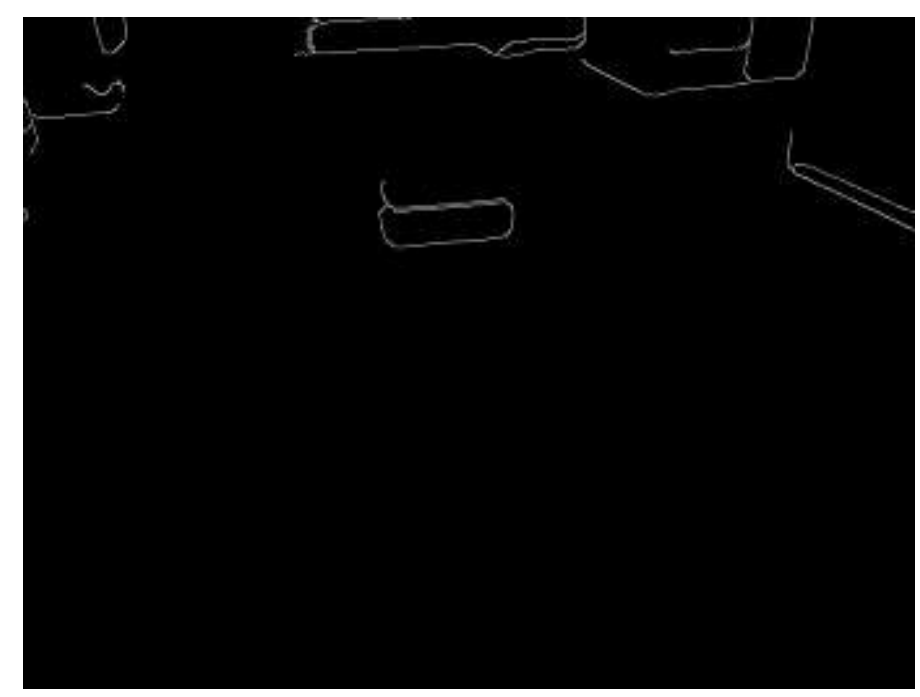

Figure 3. Canny Algorithm

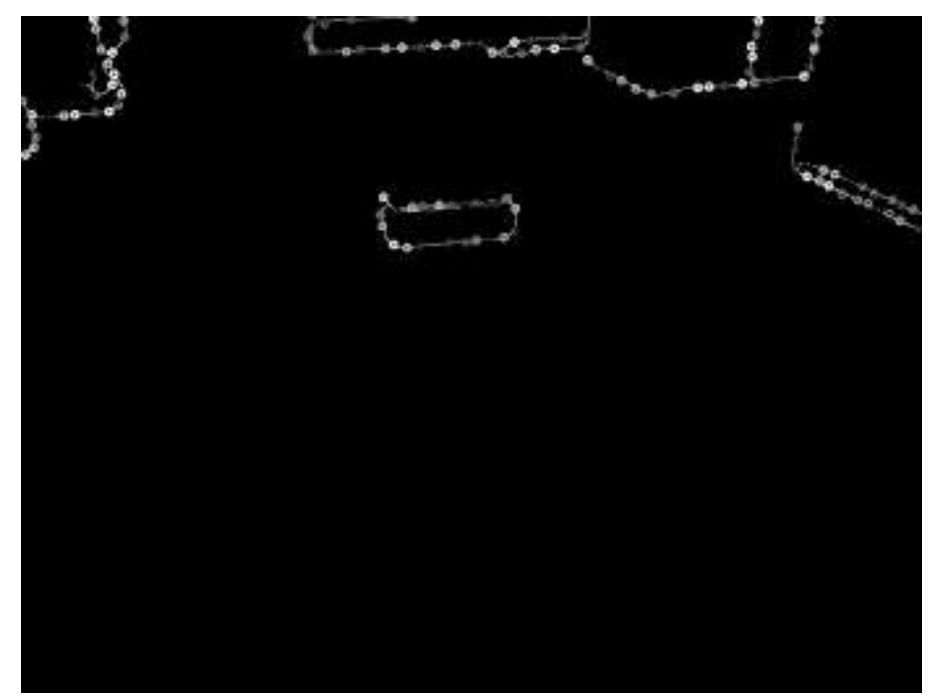

Figure 4. Edge Shi-Tomasi Corners Detection 
After image filtering and marginalized, Canny edge detection for image, it given in Figure 3, getting the edge of the obstacles or the target curve, we can select features corners and angular points which represent the local changes in the edge. It not only contains the target location and the scene to identify important information, and can achieve the accuracy of sub-pixel level, it shown in Figure 4.

In the case of slow movement of the mobile robot $(0.25 \mathrm{~m} / \mathrm{s})$, in the process of mobile robot moving, we need to find the sub-pixel coordinates of P1 corner P in the first image. Then match the sub-pixel coordinates P2 of the point P from the second image. VS2010 environment, OpenCV2.3.4 tools are used, the RANSAC matching algorithm detects points are given in Figure 5.

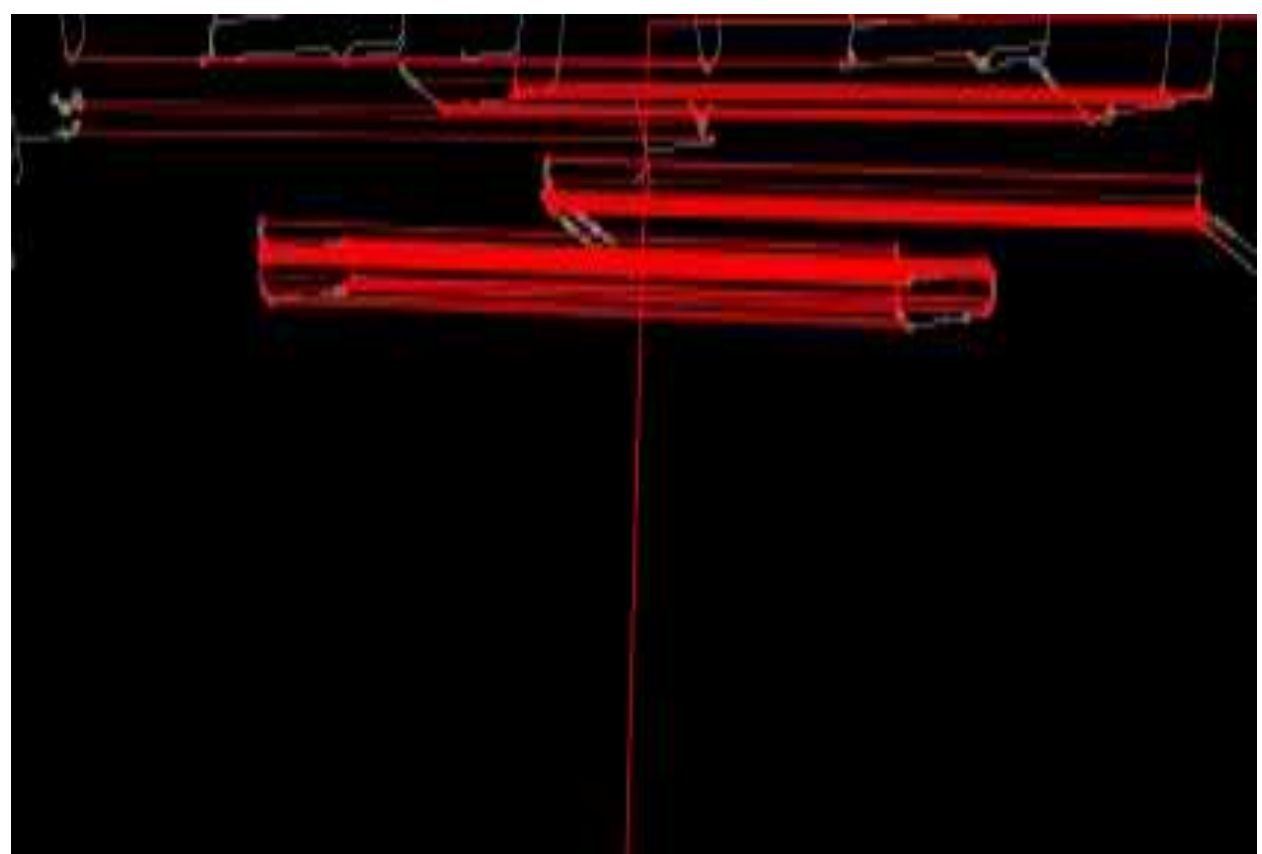

Figure 5. RANSAC Corners Matching

\section{Establish Monocular Vision Measurement Model}

\subsection{Measuring Principle}

Monocular vision is used to extract depth information of target feature points. In the laboratory environment, two important assumptions ${ }^{[10]}$ are used as constraint conditions for the depth information of feature points, also in order to meet the feasibility of the robot path planning:

1. Obstacles differ from the ground on the surface;

2. The ground is basically flat, with no impending obstacles.

These two conditions are easy to meet in the general indoor environment.

Camera is fixed on the mobile robot. There is a certain angle .././../../Administrator/AppData/Local/youdao/dict/Application/7.3.0.0817/resultui/dict/?keyword= of

....././../Administrator/AppData/Local/youdao/dict/Application/7.3.0.0817/resultui/dict/?keyword= inclination between the optical axis and the ground. This installation method can not only collect the appropriate distance of the environment, And the camera has better clarity in the field of view. It's benefit to perceive environment and build map; Optional $\mathrm{P}$ is the feature point of edge image, $\mathrm{P} 1$ and $\mathrm{P} 2$ are objects of point $\mathrm{P}$ on different images.H remarks the height of the camera, $\mathrm{M}$ for the front camera along the robot moving distance. 
The $m$ remarks the distance of the camera moving along front of the robot. Figure 6 illustrates the geometrical properties of the camera image plane along with the corresponding transformed plane. A robot acquires images 1 and 2 at two nearby times, $\theta_{1}$ and $\theta_{2}$, respectively, and can apply the relative transformation by using the odometer information between the two frames.

For the convenience of calculation, definite $2 \mathrm{a}$ as field of view in vertical direction of camera(available from experiment, $\tan (2 a)=y_{1} / H$ ), and $\mathrm{b}$ for the angle of camera installation overlooks. the number of pixels of images on the $\mathrm{Y}$ axis, the total number of pixels for pictures on the $\mathrm{X}$ axis. $S_{y}$ is the total number of $\mathrm{Y}$ axis pixels for images. $S_{x}$ is the total number of $\mathrm{Y}$ axis pixels for images.

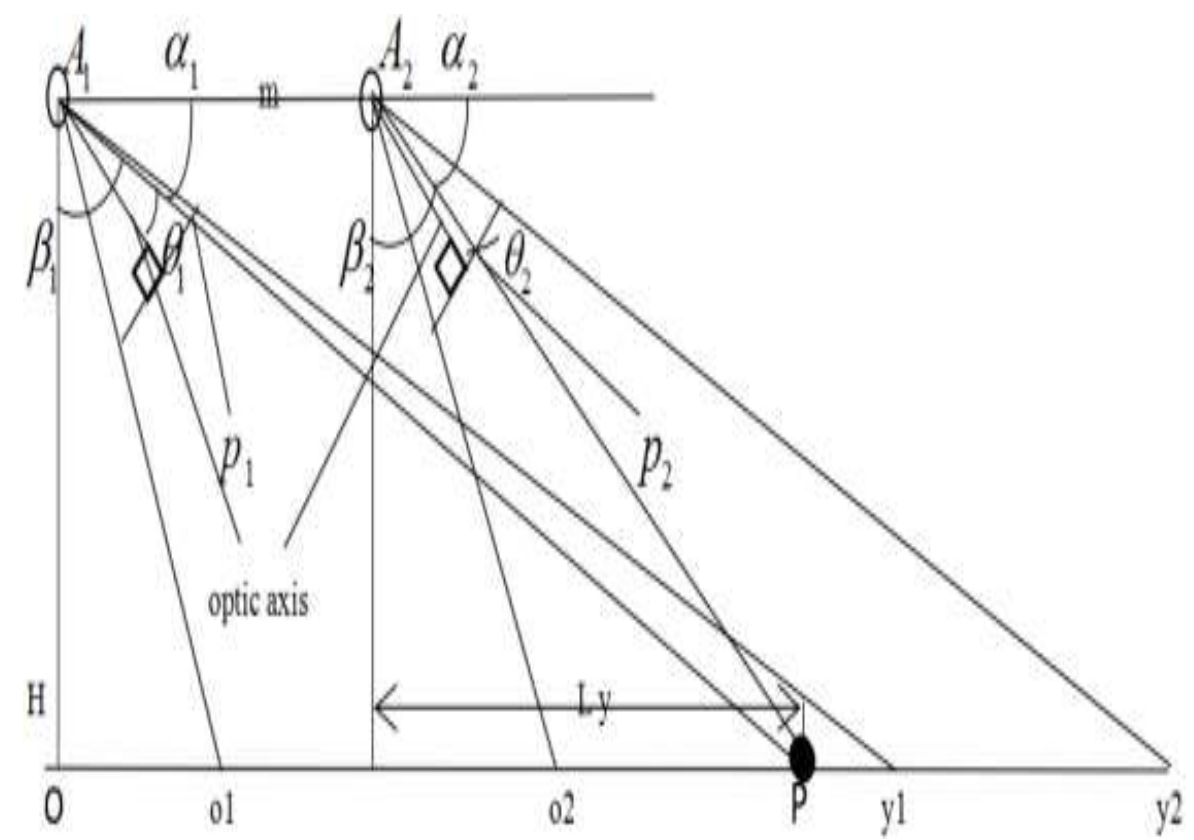

Figure 6. Horizontal Projection of Monocular Visual Measuring Model

Figure 6 geometric relationship can be solved:

$$
\begin{gathered}
\frac{\tan \theta_{1}}{\tan a}=\frac{S_{y} / 2-y_{1}}{S_{y} / 2} \\
\frac{\tan \theta_{2}}{\tan a}=\frac{S_{y} / 2-y_{2}}{S_{y} / 2} \\
\theta_{1}=\arctan \left[\frac{\left(S_{y} / 2-y_{1}\right) \times \tan a}{S_{y} / 2}\right] \\
\theta_{2}=\arctan \left[\frac{\left(S_{y} / 2-y_{2}\right) \times \tan a}{S_{y} / 2}\right] \\
\alpha_{1}=\pi / 2-\mathrm{b}-a-\theta_{1} \\
\alpha_{2}=\pi / 2-\mathrm{b}-a-\theta_{2} \\
\left(L_{y}+m\right) \times \tan \left(\alpha_{1}\right)=L_{y} \times \tan \left(\alpha_{2}\right)
\end{gathered}
$$




$$
L_{y}=\frac{m \times \tan \left(\alpha_{1}\right)}{\tan \left(\alpha_{2}\right)-\tan \left(\alpha_{1}\right)}
$$

$L_{y}$ is the straight distance between the target point and the robot.

Overlooking the monocular visual distance measurement model as shown in Figure 7, $\gamma$ is the maximum horizontal camera angle which can be measure with experiment, $\varphi$ indicates the angle between $\stackrel{\text { uw }}{O P}$ and $\stackrel{\mathrm{uu}}{O Y}$, which is demanding goal point and the distance between the origin $\mathrm{O}, \mathrm{D}$ indicates the distance between the origin of $\mathrm{O}$ and the target point, $\mathrm{O} 2$ is the closest point that the camera can see.

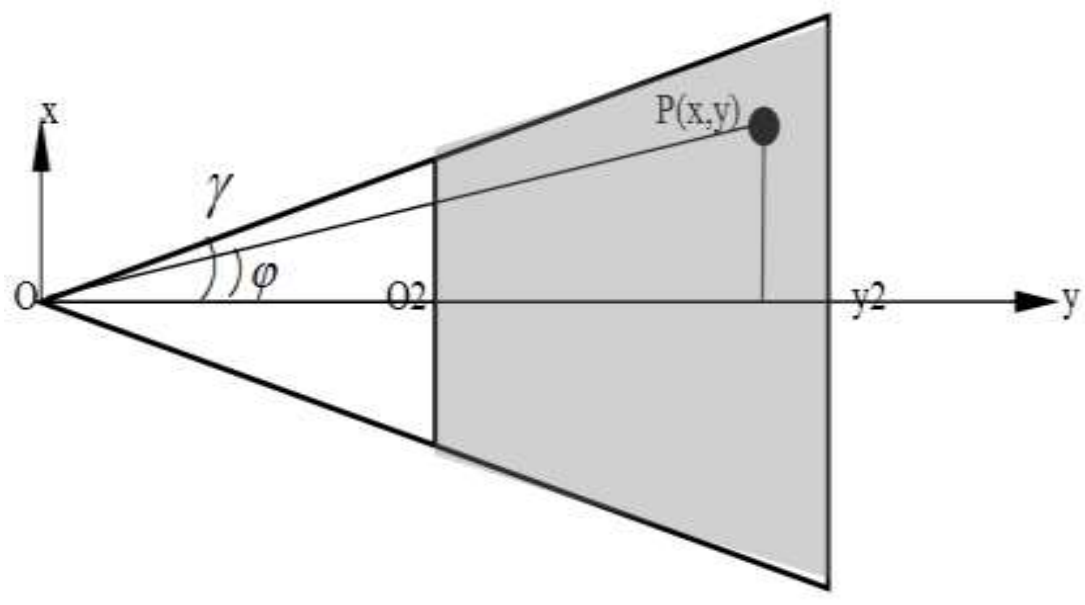

Figure 7. Vertical Projection of Measuring Model

In Figure 7, the shaded portion is the visual area of the camera. In the same way, The vertical distance from the object to the robot's forward direction is $L_{x}$. For the convenience of distinguishing measurement results, The coordinates of the target point on the left side of the camera axis (upper side of the image) is positive. The coordinates of the target point on the right side of the camera axis (below side of the figure) is negative.

$$
\begin{gathered}
\frac{\tan \varphi}{\tan \gamma}=\frac{S_{x} / 2-x_{2}}{S_{x} / 2} \\
L_{x}=L_{y} \cdot \tan \varphi \\
L_{x}=L_{y} \cdot \frac{S_{x} / 2-q_{2}}{S_{x} / 2} \cdot \tan \gamma
\end{gathered}
$$

$\mathrm{D}$ indicates the distance between the robot and the target.

$$
D=\longdiv { L _ { x } ^ { 2 } + L _ { y } ^ { 2 } }
$$

The above figure is shown that the measurement error is basically stable. and with the increasing of distance, the advantage of sub-pixel ranging method is obvious. In order to verify the accuracy of this algorithm, mobile robot move 10 centimeters along the front line. Test results are presented in Table 2, combined with the reference [5] and [6] the measurement error of the comparison of experimental data. 
Table 1. Compared with Literature in Target Points Test

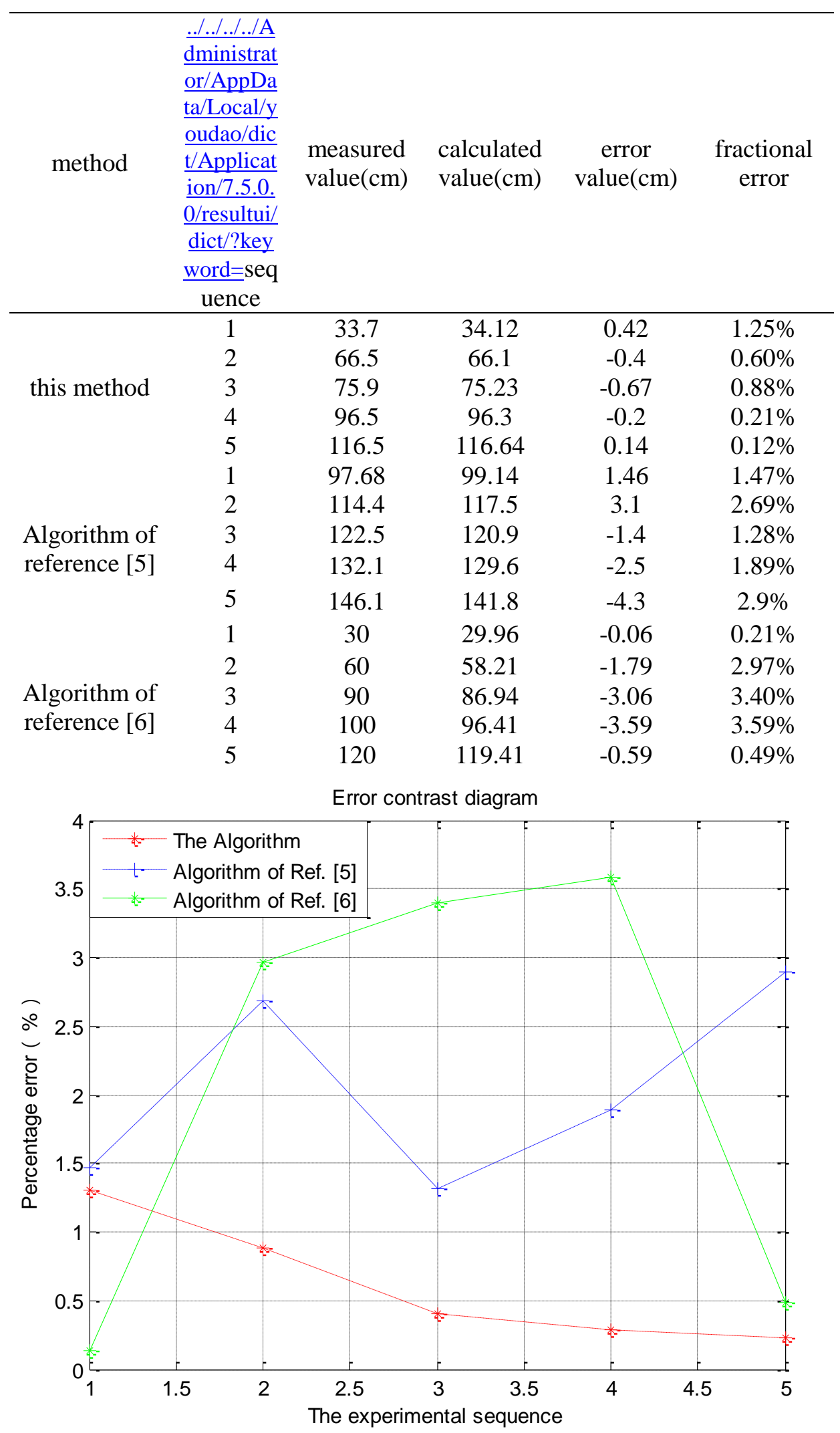

Figure 8 Relative Error Comparison 
From the data in Table 1 and Figure 8 curves can be seen. The measurement errors of literature [5] algorithm are relatively stable, but slightly higher; In the literature [6], the errors of the algorithm were unstable, and this algorithm can't guarantee to measure with high accuracy around the robot. The measuring accuracy of this algorithm is stable, and it can maintain a lower percentage of error (relative error within 1.5\%). With the increase of distance measuring advantage will be reflected, so it is helpful for a mobile robot to build environment model and make itself position.

\subsection{Analysis of Real Time Operation}

Due to the complicated indoor environment, the robot moved at a speed of $0.25 \mathrm{~m} / \mathrm{s}$, and the sampling period of the image was 0.4 second and the distance was $0.1 \mathrm{~m}$. Because of the low speed of a mobile robot, the effect of blurred image is ignored here. In order to verify the timeliness of mobile robot system's extraction and matching of image feature points, the influence of sub-pixel extraction and matching on system measurement time is analyzed.

Table 2. Computing Time of this Algorithm

\begin{tabular}{cccc}
\hline $\begin{array}{c}\text { Feature } \\
\text { points }\end{array}$ & $\begin{array}{c}\text { Matching number } \\
\text { of feature points }\end{array}$ & $\begin{array}{c}\text { Pixel(second } \\
\text { ) }\end{array}$ & $\begin{array}{c}\text { Sub- } \\
\text { pixel(second) }\end{array}$ \\
\hline 230 & 120 & 0.288 & 0.305 \\
230 & 90 & 0.260 & 0.275 \\
230 & 60 & 0.230 & 0.236 \\
230 & 30 & 0.199 & 0.207
\end{tabular}

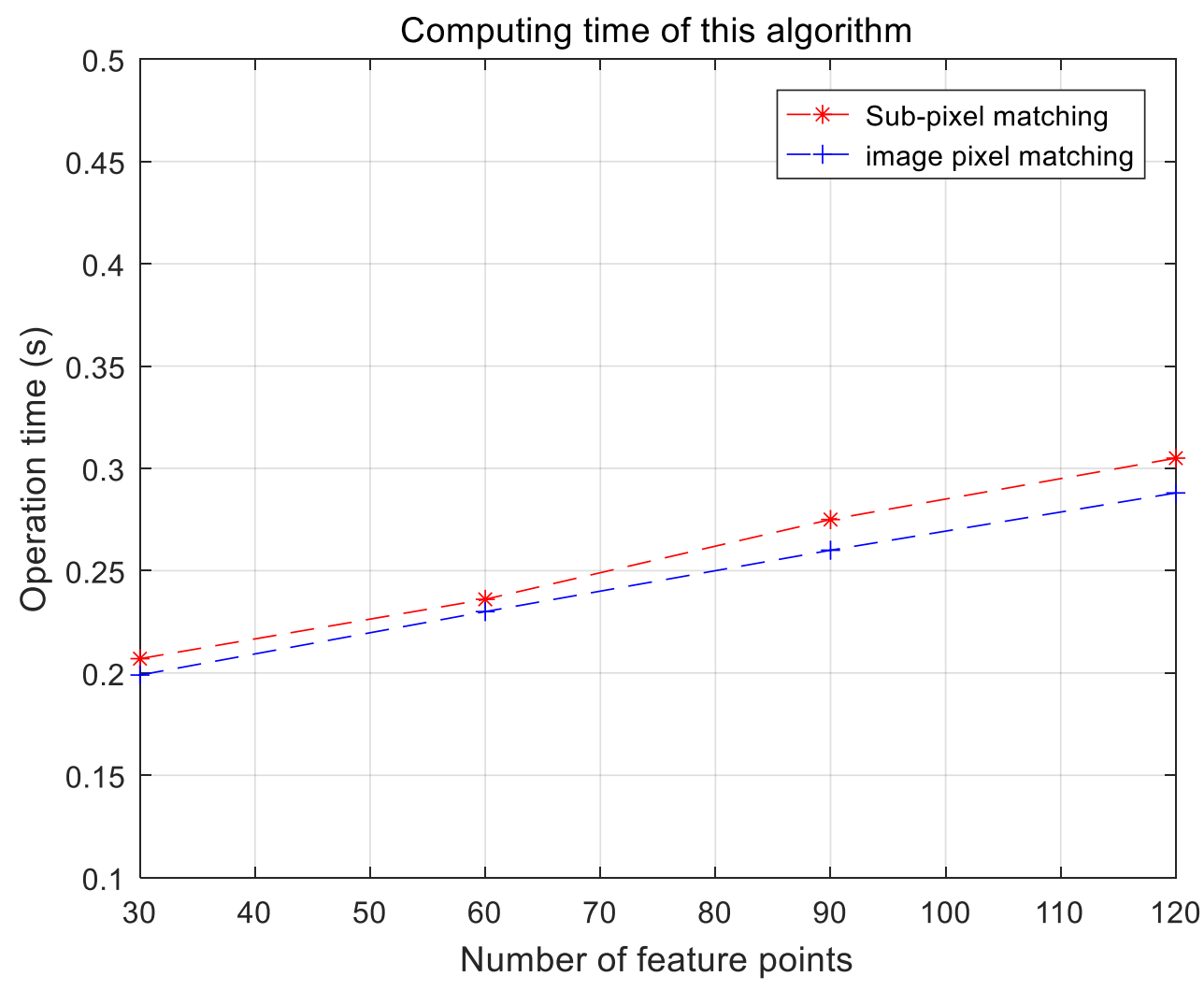

Figure 9. Operation Time Comparison 
The Table 2 and Figure 9 shows that the using time of sub-pixel feature points detection and matching is more than the pixel feature points, but the calculation time is less than the sampling time ( 0.4 second) algorithm, the algorithm can achieve positioning and meet the timeliness of algorithm for mobile robot.

\section{Measurement of Target Positioning}

To calculate the location of target $\mathrm{P}_{2}$, we can set up the camera coordinate system $O_{2}-X_{c} Y_{c} Z_{c}$ and the mobile robot coordinate $O_{2}-X_{r} Y_{r} Z_{r}$. Coordinate of the imaging target point $\mathrm{P} 2$ is $\left(x_{2}, y_{2}, f\right)$, as shown in Figure 10.

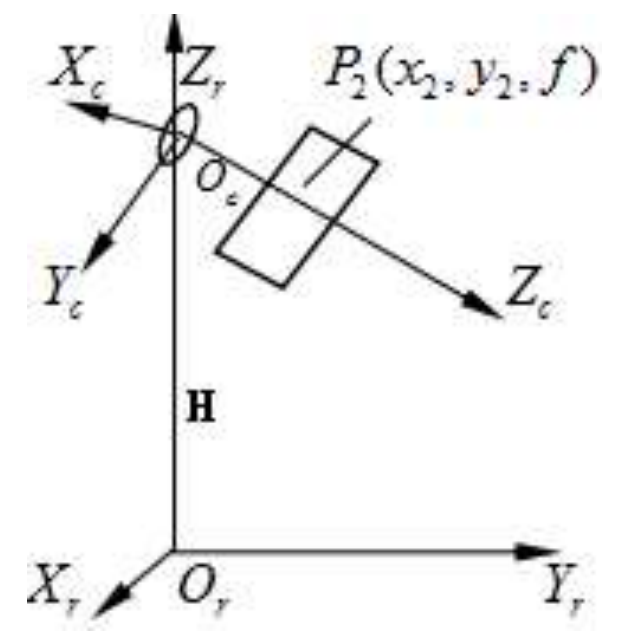

Figure10. Establish Coordinate

By combining the Figure 6 and Figure 10, the mobile robot coordinate system can be gotten from the camera coordinate system with $(a+b)$ rotating angle around the $\mathrm{X}$ axis, the location of the measuring points can be achieved according to the transformation between coordinate systems. The homogeneous coordinate relationship between the mobile robot coordinate system and the camera coordinate system can be expressed as:

$$
\begin{aligned}
& {\left[\begin{array}{c}
X_{r} \\
Y_{r} \\
Z_{r} \\
1
\end{array}\right]=\left[\begin{array}{cccc}
1 & 0 & 0 & 0 \\
0 & \cos (a+b) & -\sin (a+b) & 0 \\
0 & \sin (a+b) & \cos (a+b) & -H \\
0 & 0 & 0 & 1
\end{array}\right]\left[\begin{array}{c}
X_{c} \\
Y_{c} \\
Z_{c} \\
1
\end{array}\right]} \\
& \left\{\begin{array}{c}
X_{r}=X_{c} \\
Y_{r}=Y_{c} \cos (a+b)-Z_{c} \sin (a+b) \\
Z_{r}=Y_{c} \sin (a+b)+Z_{c} \cos (a+b)-H \\
1=1
\end{array}\right.
\end{aligned}
$$

Formula (1) to (14) can get the coordinates and direction of the target point P2 in the mobile robot coordinate system,

$$
\begin{gathered}
L_{y}=\frac{m \times \tan \left(a+b+\theta_{2}\right)}{\tan \left(a+b+\theta_{1}\right)-\tan \left(a+b+\theta_{2}\right)} \\
L_{x}=L_{y} \cdot \frac{S_{x} / 2-q_{2}}{S_{x} / 2} \cdot \tan \gamma
\end{gathered}
$$


The distance is shown in formula (12), $\varphi$ indicates that the target of $\mathrm{P}$ and mobile robot coordinate origin $O_{r}$ attachment and advance angle direction .

$$
\varphi=\arctan \left(\frac{Y_{r}}{X_{r}}\right)
$$

In the practical application of the mobile robot, the distance and direction of all extraction points are obtained after obtaining all key points. In order to further reduce the processing time, with minimal feature points instead of the most important environmental information, so we need to match the results after the filtrated, the filtrating specific rules will discuss in the follow-up work.

\section{Conclusion}

the improved based on monocular vision ranging of sub-pixel image processing model, simplified single visual distance model, and through the edge detection, sub-pixel corner extraction and matching, combined with the movement parameters of the mobile robot, the information between the mobile robot and the obstacle could be got. With the proposed method, the target was located at different locations. That the measurement accuracy and effectiveness of the method can meet, the experimental results show the demand for avoidance.

\section{Acknowledgments}

This work is supported by intelligent signal and information processing sichuan youth science and technology innovation research team (2015TD0022), Key research and development projects of Sichuan province's first batch of scientific and technological plans in 2017(2017GZ0068). Graduate innovation fund project of Sichuan University of Science and Engineering (y2016040).

\section{References}

[1] X. Shao and J. W. Tao, "A method of stationary target localization based on monocular vision", Acta Photonica Sinica, vol. 10. 45, no. 10, (2016), pp. 128-135.

[2] G. Wu and Z. M. Tang, "Study on the ranging of monocular autonomous robot vision navigation", Robot, vol. 32, no. 6, (2010), pp. 828-832.

[3] C. J. Zhang, S. J. Ji and N. F. Xiao, "Distance measurement based on monocular vision", Journal of Shandong University of Science and Technology, vol. 26, no. 4, (2007), pp. 65-68.

[4] X. Liu, Q. Tian and W. Chen, "Real-time distance measurement using a modified camera", Sensors Applications Symposium,USA: IEEE, (2010), pp. 54-58.

[5] L. Qiang, "Research on monocular vision real-time ranging algorithm", Harbin Institute of Technology, (2014).

[6] 6. Y. P. Li, "Research on robot ranging based on machine vision", sensor world, no. 4, (2014), pp. 7-11.

[7] L. Guo and Y. Xu, "Research on real time range finding method based on monocular vision", Chinese Journal of image and graphics, vol. 11, no. 1, (2006), pp. 74-81.

[8] J. B. Lin, C. Y. Su, J. B. Zheng and X. Y. Yu, "Machine vision guided vehicle indoor automatic positioning system", mechanical science and technology, vol. 34, no. 11, (2015), pp. 1675-1681.

[9] C. C. Hsu, M. C. Lu and K. W. Chin, "Distance measurement based on pixel variation of CCD images", ISA Transactions, vol. 48, no. 4, (2009), pp. 389-395.

[10] R. Siegwart and R. Illah, "Introduction to Autonomous Mobile Robots (Second Edition)", Li R H, Song Q S, Xi'an: Xi'an Jiao Tong University press, (2013).

[11] W. B. Wang, "Research on real time ranging method based on monocular vision", Dalian University of Technology, (2014).

[12] J. Cui, J. Huo and M. Yang, "Research on the rigid body posture measurement using monocular vision by coplanar feature points", Optik - International Journal for Light and Electron Optics, vol. 126, no. 24, (2015), pp. 5423-5429.

[13] F. Gao, X. Ma, J. Gu, "An active target localization with monocular vision", IEEE International Conference on Control \& Automation. IEEE, (2014), pp. 1381-1386.

[14] J. C. Ren and X. D. Yang, "Research on high precision binocular vision ranging based on mutual information", control engineering, vol. 22, no. 1, (2015), pp. 199-204. 
[15] Q. C. Sun, Y. Z. Zhou and C. Ning, "Subpixel edge detection method based on three spline interpolation", control engineering, vol. 21, no. 2, (2014).

[16] M. C. Lu, C. C. Hsu and Y. Y. Lu, "Distance and angle measurement of distant objects on an oblique plane based on pixel variation of CCD image", Instrumentation and Measurement Technology Conference (I2MTC), 2010 IEEE. IEEE, (2010), pp. 318 - 322.

[17] B. Li, X. Zhang and M. Sato, "Pitch Angle Estimation Using a Vehicle Mounted Monocular Camera for Vehicle Target Range Measurement", Journal of the Institute of Image Information \& Television Engineers, vol. 69, no. 4, (2015), pp. J169-J176.

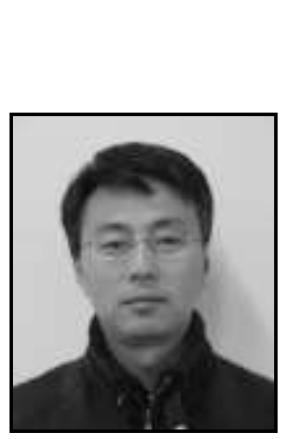

\section{Authors}

Xiaohong Ren, he was born in 1960, M. S., professor, comes from School of Automation and Information Engineering, Sichuan University of Science and Engineering. Corresponding Author, Email: rx88@163.com, His research interests in the teaching and research of information processing and intelligent control.

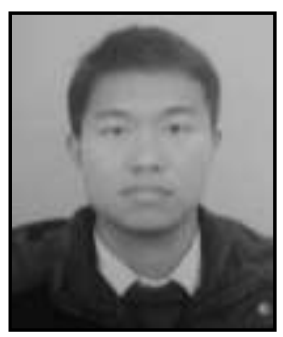

Zhengguang Zheng, he was born in 1990, M. S. candidate, comes from School of Automation and Information Engineering, Sichuan University of Science and Engineering. His research interests include robot control and machine vision.

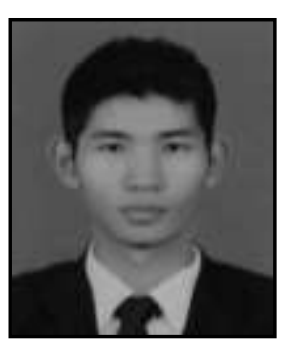

Yajun Cheng, he was born in 1992, M. S. candidate, comes from School of Automation and Information Engineering, Sichuan University of Science and Engineering. His research interests include robot control and machine vision. 
International Journal of Control and Automation

Vol. 11, No. 3 (2018) 